\title{
Caractérisation expérimentale des transferts couplés de chaleur et de masse dans les enveloppes de constructions en bois
}

\author{
Helisoa Rafidiarison ${ }^{1,2}$, Éric Mougel $^{1, a}$ ET Alexis Nicolas $^{2,3}$ \\ 1 Université de Lorraine, LERMAB, ENSTIB, 27 rue Philippe Séguin, 88051 Épinal, France \\ 2 CRITT BOIS, 27 rue Philippe Séguin, 88051 Épinal, France \\ 3 AVEN'R, 2T grande rue, 88460 Faucompierre, France
}

Reçu le 17 Avril 2012, accepté le 29 Octobre 2012

Résumé - Une double enceinte climatique conçue sur le principe de la méthode de la boîte chaude gardée a été mise au point afin d'étudier en laboratoire le comportement thermique et hygrique de différentes configurations de parois en bois. Le dispositif est doté d'équipements de régulation lui permettant de reproduire des sollicitations climatiques stationnaires ou dynamiques proches d'un climat réel et de suivre l'évolution des profils de température et d'humidité dans les parois testées. Dans ce travail, le suivi du comportement hygrothermique d'une paroi en ossature bois comportant des matériaux hygroscopiques et soumises à différentes conditions est rapporté. Les résultats ont montré que la capacité du dispositif à bien reproduire les conditions imposées a permis de mesurer les propriétés importantes de parois en régime permanent (résistance thermique) et transitoire (amortissement et déphasage thermique). Les essais réalisés ont également mis en évidence le comportement hygroscopique des matériaux testés.

\begin{abstract}
Mots clés : Transferts couplés chaleur-masse / construction bois / dispositif expérimental / matériaux hygroscopiques
\end{abstract}

\begin{abstract}
Experimental investigation of coupled heat and mass transfer in wooden building envelopes. A double climatic chamber based on the guarded hot box method was designed to study the hygrothermal behaviour of different compositions of timber walls. Equipment installed allows to reproduce in the climatic chamber hygrothermal conditions close to real climatic conditions and to assess the evolution of temperature and humidity profiles of the tested walls. This paper deals with the hygrothermal behaviour of a timber-framed wall including hygroscopic materials. The results showed that the ability of the experimental device to reproduce desired conditions made it possible to measure wall thermal properties during steady-state (thermal resistance) and transient conditions (decrement factor and time lag). The hygroscopic behaviour of tested materials was also observed during the tests.
\end{abstract}

Key words: Coupled heat and mass transfer / wooden construction / experimental apparatus / hygroscopic materials

\section{Introduction}

Ces dernières décennies, l'intérêt croissant du secteur de la construction pour un bâtiment confortable, plus performant énergétiquement et à faible impact environnemental a favorisé l'utilisation du bois et des matériaux bio-sourcés en général. En effet, de précédents travaux [1-7] ont montré que les transferts couplés de chaleur et de masse dans le bois et d'autres matériaux hygroscopiques peuvent contribuer au confort intérieur et à la performance énergétique des constructions.

\footnotetext{
a Auteur pour correspondance :

eric.mougel@enstib.uhp-nancy.fr
}

D'une part, la particularité des matériaux hygroscopiques consiste en leur capacité à se mettre en équilibre thermohygrique avec le milieu environnant. Pendant ce processus et selon le taux d'humidité de l'air ambiant, le matériau peut absorber ou restituer de l'humidité à l'air. Les mêmes études ont ainsi montré l'intérêt de cette propriété des matériaux hygroscopiques dans la performance globale de l'enveloppe. D'autre part, ces matériaux contribuent également au confort thermique intérieur car ils amortissent les fluctuations de température extérieures et apportent un déphasage intéressant des ondes de chaleur extérieure, assurant ainsi un intérieur quasi-stable en température. Ces propriétés ont déjà été mesurées à 
l'échelle du matériau [8,9], mais à l'échelle de la paroi, leur détermination se limite souvent à une estimation analytique en régime permanent et basée sur des paramètres souvent considérés comme constants. Pourtant, le comportement réel de ces matériaux pourrait différer des calculs analytiques du fait de la non prise en compte dans les calculs de propriétés ou phénomènes intervenant dans les transferts dans la paroi, ce qui rend nécessaire la réalisation d'études numériques ou expérimentales du comportement de ces matériaux à l'échelle paroi pour mieux comprendre les processus impliqués dans ces différents comportements.

Des dispositifs expérimentaux existent déjà et sont utilisés pour caractériser le comportement de parois. Le plus souvent, ces dispositifs sont basés sur la méthode de la boîte chaude gardée et consistent en la mesure de propriétés thermiques dynamiques ou stationnaires des parois [10-12]. D'autres études, traitant du comportement hygrothermique des parois, ont également utilisé des dispositifs expérimentaux similaires en adaptant une régulation d'humidité au dispositif de base $[13,14]$.

Dans le travail présenté ici, nous nous proposons d'utiliser une double enceinte climatique pour caractériser expérimentalement les transferts couplés de chaleur et d'humidité à travers différentes parois de constructions bois, en conditions stationnaires et dynamiques. Dans un premier temps, une description détaillée du dispositif expérimental est donnée. Ensuite les essais réalisés sur les parois testées seront abordés et nous terminons par une analyse et discussion des résultats obtenus.

\section{Dispositif expérimental}

\subsection{Double enceinte climatique}

Afin de caractériser le comportement thermique et hydrique en régimes transitoire et stationnaire de différentes configurations de parois en bois à échelle réelle, une double enceinte climatique a été conçue et construite sur le site de l'ENSTIB à Épinal. La conception du dispositif est en partie basée sur la méthode de la boîte chaude gardée décrite dans la norme EN ISO 8990 [15] qui permet la détermination expérimentale des propriétés de transfert de chaleur en régime permanent. Le dispositif type «boîte chaude » a été adaptée afin de mesurer également l'évolution temporelle des profils de température et d'humidité de la paroi testée.

Le dispositif est composé de deux caissons (intérieur et extérieur), séparés par la paroi testée. Les deux caissons sont dotés chacun d'un équipement de régulation assurant le chauffage, le refroidissement, l'humidification et la déshumidification de l'air ambiant. Ce système couplé à un système de pilotage de la régulation permet de reproduire dans les deux caissons des conditions stationnaires ou dynamiques en température et humidité proches des sollicitations réelles intérieure et extérieure d'un bâtiment. Afin de limiter les échanges avec l'extérieur de la double enceinte climatique, les caissons sont isolés avec $120 \mathrm{~mm}$ de polyuréthane et rendus étanches à l'air et à la vapeur d'eau. Les dimensions de chaque caisson sont de 2,8 $\mathrm{m}$ de largeur, $3 \mathrm{~m}$ de longeur et $2,8 \mathrm{~m}$ de hauteur. Il est donc possible de tester des parois de $2,8 \mathrm{~m}$ de large et $2,8 \mathrm{~m}$ de haut avec une épaisseur maximale de $50 \mathrm{~cm}$.

Le caisson intérieur, conçu pour reproduire les conditions intérieures d'un bâtiment, possède une centrale de traitement d'air installée à l'arrière du caisson. Un compresseur installé à l'extérieur du caisson et relié à la centrale de traitement d'air, constitue l'unité de refroidissement et de déshumidification de l'air. Une résistance électrique de $2500 \mathrm{~W}$ est installée en sortie de la centrale de traitement d'air pour chauffer l'air sortant. Le processus d'humidification se fait par le biais d'un système de vaporisation d'eau comprenant une résistance électrique de $250 \mathrm{~W}$. Ces équipements permettent de simuler des conditions de température intérieure entre 0 et $30^{\circ} \mathrm{C}$ avec une humidité relative de l'air allant de 30 à $95 \%$.

Le système de traitement d'air du caisson extérieur est similaire à celui décrit auparavant. Pour simuler des sollicitations climatiques extérieures, des équipements de chauffage et de refroidissement plus puissants ont été installés. Ainsi, la résistance de chauffe a une puissance de $4000 \mathrm{~W}$ (ce qui permet d'atteindre une température au moins égale à $45{ }^{\circ} \mathrm{C}$ ) et le groupe froid a été dimensionné pour permettre de descendre à une température d'air négative, allant jusqu'à $-15{ }^{\circ} \mathrm{C}$. L'ensemble permet donc de contrôler une température d'air comprise entre -15 et $45^{\circ} \mathrm{C}$ avec une humidité relative de 30 à $95 \%$.

Sur la base du dispositif boîte chaude gardée, un caisson de mesure de dimensions $1,4 \mathrm{~m} \times 0,65 \mathrm{~m} \times 1,4 \mathrm{~m}$ a été construit et utilisé pour la mesure du flux de chaleur traversant la paroi testée et de son coefficient de transmission thermique. Afin de limiter les flux parasites à travers les parois du caisson de mesure, ce dernier est installé dans le caisson intérieur et régulé à la même température grâce à une résistance électrique de $55 \mathrm{~W}$. La régulation en humidité y est assurée par une solution saline saturée. Les parois du caisson de mesure sont en polyuréthane (60 mm) rendu étanche à l'air et à la vapeur d'eau.

\subsection{Métrologie}

Pour déterminer le comportement de la paroi testée, lors de l'essai, différents éléments de mesure sont installés dans le dispositif expérimental.

Pour assurer le bon fonctionnement de la régulation et pour vérifier l'homogénéité du flux d'air dans les caissons intérieur et extérieur, les distributions de température et d'humidité relative de l'air sont mesurées à l'aide de neuf thermohygromètres Sensirion SHT75 installés à $150 \mathrm{~mm}$ de la surface de la paroi étudiée.

La mesure de la température de surface est réalisée à l'aide de neuf thermocouples de type $\mathrm{T}$ d'un diamètre de 0,25 mm (conseillé dans la norme EN ISO 8990). Des thermohygromètres de type SHT 75 installés à côté de chaque thermocouple de surface permettent de suivre l'évolution de l'humidité relative de l'air proche de la surface.

Le suivi de la réponse de la paroi testée aux sollicitations imposées est réalisé à l'aide de capteurs SHT 75. Ces sondes sont installées à différentes épaisseurs des éléments 


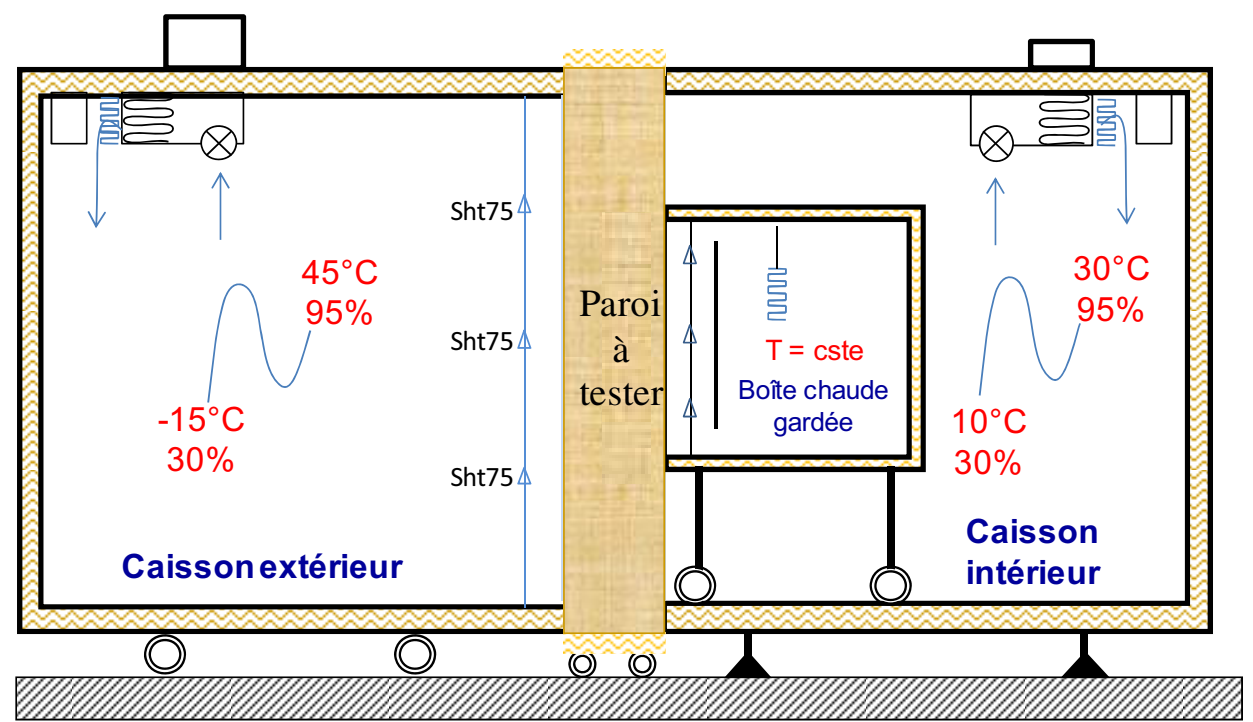

Fig. 1. Schéma de principe de la double enceinte climatique.

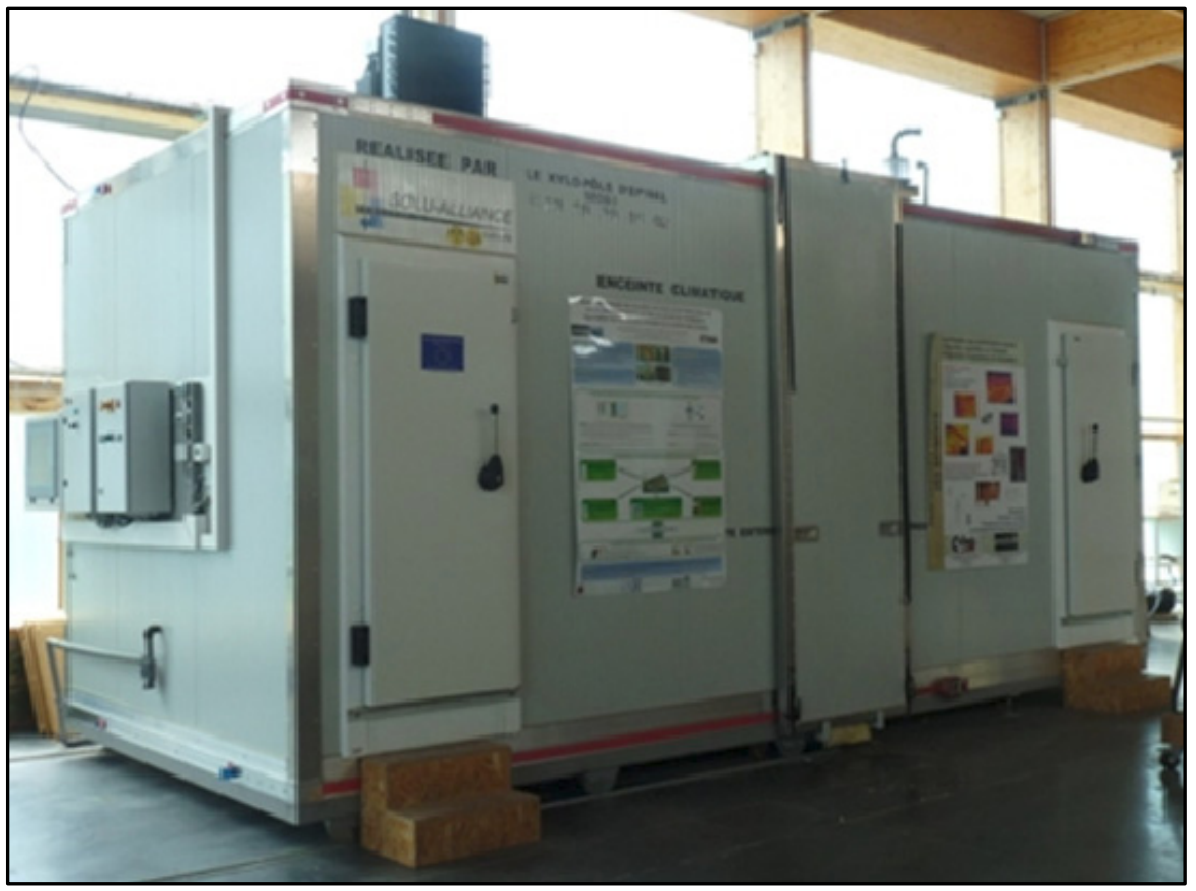

Fig. 2. Vue d'ensemble de la double enceinte climatique.

de la paroi, permettant ainsi le suivi de l'évolution des profils de température et d'humidité de la paroi. Pour la mesure de l'humidité des matériaux : le capteur SHT 75 mesure la température et l'humidité relative de l'air autour du capteur, ce qui permet de déduire son humidité absolue. Le matériau est supposé être localement en équilibre avec l'air qui l'entoure, il est alors possible d'obtenir la teneur en eau des différents matériaux qui constituent la paroi.

L'acquisition des données issues des différents capteurs est assurée par une centrale d'acquisition comprenant pour l'ensemble du dispositif : 32 voies thermocouples, 124 voies pour les sondes SHT 75 (température et humidité relative), 64 voies analogiques, 12 voies de comptage d'énergie et 8 entrées/sorties relais.

\section{Procédure expérimentale}

La paroi testée dans l'étude présentée ici, est une ossature en bois de 45/200 dont la composition, de l'extérieur vers l'intérieur, est comme suit : bardage $21 \mathrm{~mm} /$ lame d'air $25 \mathrm{~mm} /$ fibre de bois rigide $35 \mathrm{~mm}$ (FB rigide)/isolant fibre de bois souple $200 \mathrm{~mm}$ (FB souple)/panneau MFP $12 \mathrm{~mm} /$ vide technique $40 \mathrm{~mm} /$ plaque de plâtre + cellulose $13 \mathrm{~mm}$. Une 


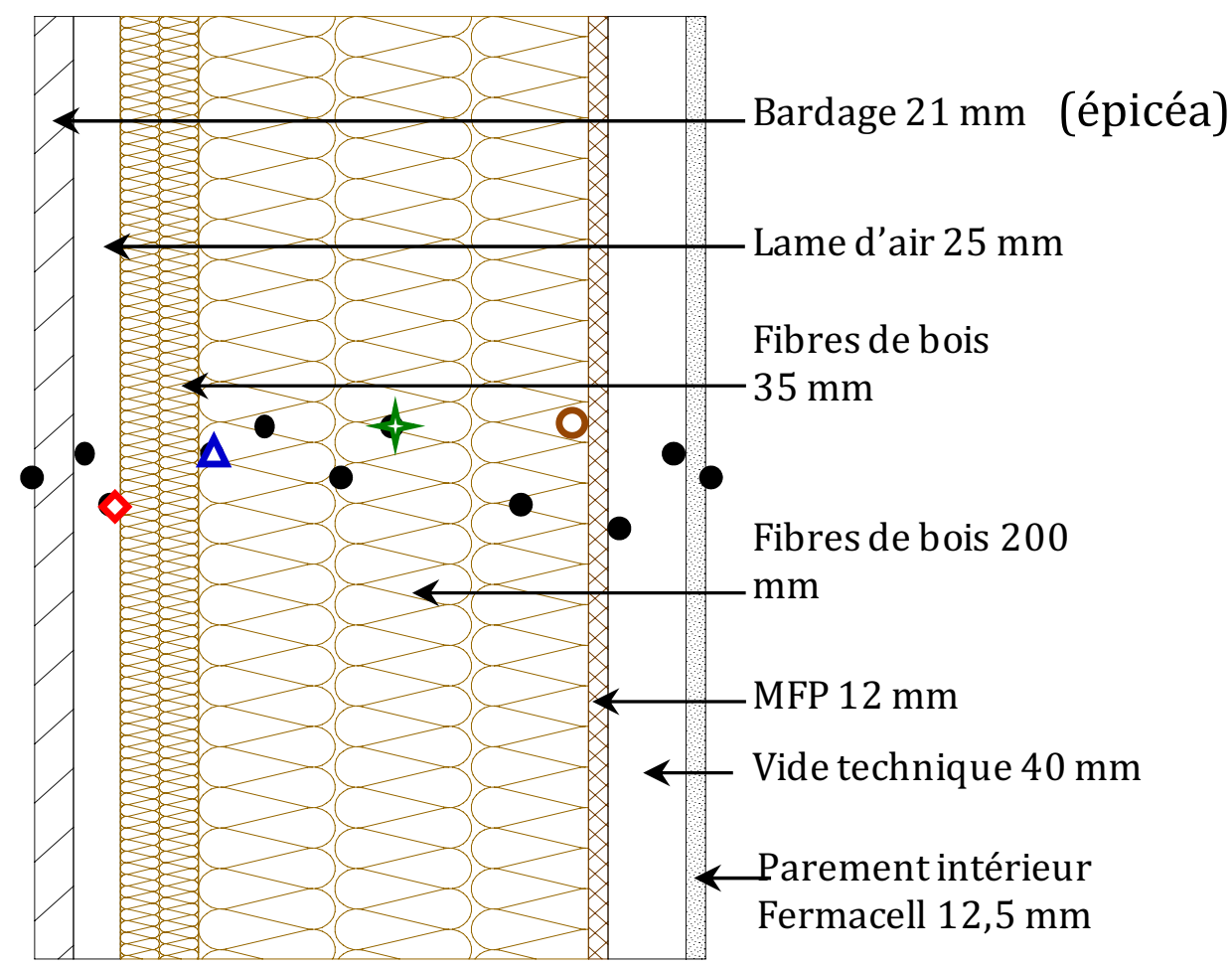

Fig. 3. Partie courante de la paroi testée et emplacement des capteurs.

représentation schématique de la partie courante de la paroi est donnée sur la figure 3.

Dans un premier temps, des essais en conditions stationnaires ont été réalisés pour mesurer la résistance thermique des parois. Les conditions d'essais ont été choisies suivant les exigences de la norme EN ISO 8990 (1996) pour la mesure des propriétés thermiques d'une paroi. Afin de mesurer la résistance thermique dans des conditions réalistes, les parois ont donc été exposées à des conditions intérieures de $25{ }^{\circ} \mathrm{C}$ et $40 \%$ d'humidité relative et des conditions extérieures hivernales de $5{ }^{\circ} \mathrm{C}$ et $80 \%$ d'humidité relative. Les parois sont ensuite mises en conditionnement thermique sous $20{ }^{\circ} \mathrm{C}-40 \%$ puis des conditions dynamiques estivales de 4 jours puis hivernales de 7 jours ont été imposées dans le côté extérieur tandis que le côté intérieur était maintenu à $20{ }^{\circ} \mathrm{C} 40 \%$. Les conditions extérieures utilisées sont celles enregistrées dans les Vosges pendant l'été et l'hiver 2010.

\section{Résultats}

La résistance thermique mesurée pour la paroi en ossature est de $5,56 \mathrm{~m}^{2} . \mathrm{K} . \mathrm{W}^{-1}$. En utilisant l'équation (1) la valeur obtenue par le calcul est de $6,21 \mathrm{~m}^{2} . \mathrm{K} \mathrm{W}^{-1}$. On note une sous-estimation de $11 \%$ de la mesure.

$$
R_{\text {paroi }}=\frac{1}{U_{\text {paroi }}}=\frac{1}{x_{i} U_{i}+x_{j} U_{j}}=\frac{1}{x_{i} \cdot \frac{1}{\sum \frac{e_{i}}{\lambda_{i}}}+x_{j} \frac{1}{\sum \frac{e_{j}}{\lambda_{j}}}}
$$

où :

$R$ : résistance thermique $\left(\mathrm{m}^{2} . \mathrm{K} . \mathrm{W}^{-1}\right)$

$U$ : coefficient de transmission thermique $\left(\mathrm{W} \cdot \mathrm{m}^{-2} \cdot \mathrm{K}^{-1}\right.$ ) $x_{i}$ : fraction surfacique de la partie courante de la paroi (-) $x_{j}$ : fraction surfacique de la partie avec montant de la paroi $(-)$

$e_{i}$ : épaisseur des matériaux de la partie courante de la paroi $(\mathrm{m})$

$e_{j}$ : épaisseur des matériaux de la partie avec montant de la paroi $(\mathrm{m})$

$\lambda_{i}$ : conductivité thermique des matériaux de la partie courante de la paroi $\left(\mathrm{W} . \mathrm{m}^{-1} \cdot \mathrm{K}^{-1}\right)$

$\lambda_{j}$ : conductivité thermique des matériaux de la partie avec montant de la paroi $\left(\mathrm{W} \cdot \mathrm{m}^{-1} \cdot \mathrm{K}^{-1}\right)$

$i$ : désigne la partie courante (ou les matériaux de cette partie) de la paroi

$j$ : désigne la partie avec montant (ou les matériaux de cette partie) de la paroi.

La figure 4 illustre un exemple d'évolution du profil de température pendant l'essai en conditions estivales (cas d'une paroi en ossature). La dynamique de l'évolution des températures dans la paroi permet d'observer le temps mis par les pics de température extérieure pour atteindre les interfaces entre les matériaux et d'en déduire le déphasage thermique des ondes de chaleur extérieure apporté par chaque matériau. Une épaisseur de $35 \mathrm{~mm}$ de fibre de bois rigide apporte ainsi $2 \mathrm{~h}$ de déphasage tandis que la fibre de bois souple apporte autant de déphasage pour $100 \mathrm{~mm}$ d'épaisseur. 


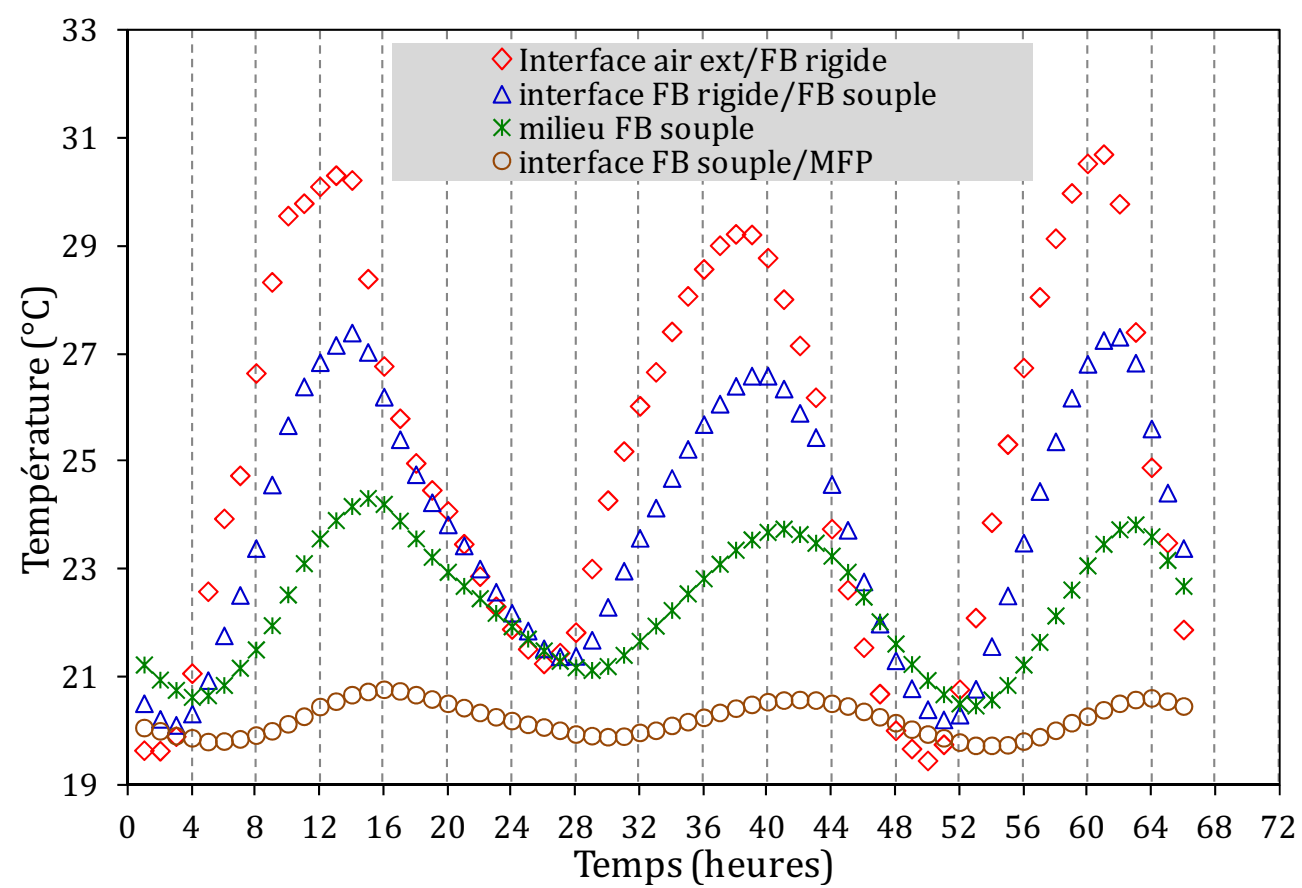

Fig. 4. Évolution du profil de température dans la paroi en ossature (l'emplacement des points est représenté sur la Fig. 3).

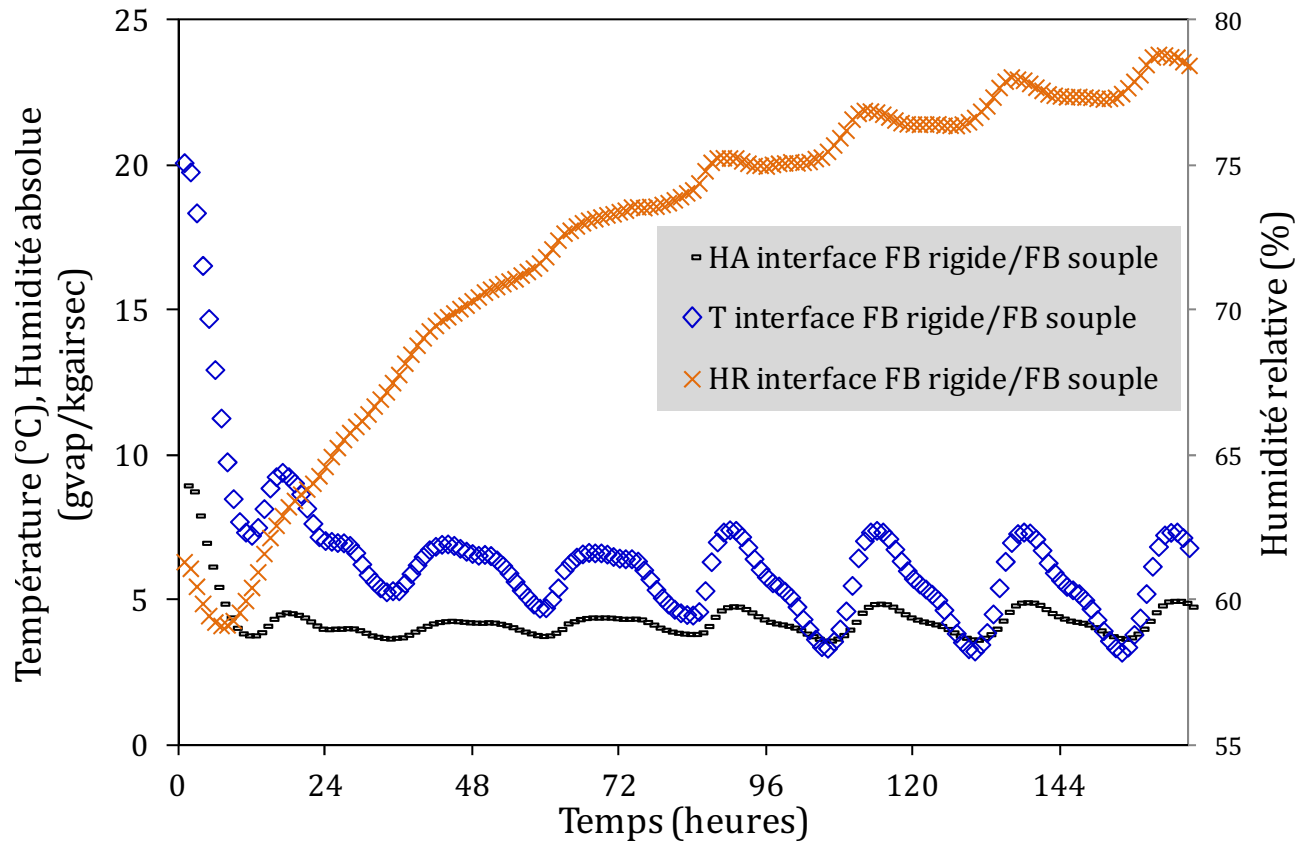

Fig. 5. Évolution de la température, de l'humidité relative et de l'humidité absolue en hiver.

Lors de l'essai où la paroi en ossature, préalablement conditionnée à $20^{\circ} \mathrm{C}$, subit une variation hivernale de la température extérieure, nous observons sur la figure 5 que la paroi subit d'abord une brusque decroissance de la température. À l'interface de l'isolant en fibre de bois rigide et de celui en fibre de bois souple, cette évolution s'accompagne d'une diminution de l'humidité relative également. Pour un matériau non hygroscopique initialement à $20^{\circ} \mathrm{C}$, une telle baisse de la température s'accompagne d'une augmentation de l'humidité relative au point de provoquer une condensation. Dans le cas de cette paroi, ce point de condensation n'est pas atteint pendant toute la durée de l'essai. Les deux isolants fibreux étant des matériaux hygroscopiques, leur teneur en eau dépend donc de l'humidité relative de l'air ambiant. Lorsque la température diminue, l'humidité relative de l'air augmente tout d'abord, et pour assurer l'équilibre de sorption, le matériau va adsorber le surplus d'humidité, ce qui entraîne la baisse d'humidité absolue de l'air environnant. Une augmentation de température entraîne 
l'effet inverse ce qui explique la dépendance de l'évolution de l'humidité absolue de l'air avec celle de la température pendant tout l'essai.

L'humidité relative a été mise en axe secondaire sur la figure 5 pour plus de lisibilité des résultats.

\section{Conclusion}

Cette étude a concerné la conception et le développement d'une double enceinte climatique permettant de tester à des conditions stationnaires ou dynamiques le comportement d'une paroi à échelle réelle. Les essais réalisés ont pu montrer la capacité de la double enceinte à caractériser les propriétés d'une paroi en température et en humidité, aussi bien en régime permanent (résistance thermique) qu'en régime transitoire. En été, le suivi du profil de température a permis d'obtenir le déphasage thermique et l'amortissement des ondes de chaleur apportés par chaque matériau. En hiver, le comportement hygroscopique des matériaux d'isolation a permis d'éviter la condensation lors d'une baisse brutale de la température. Dans ce travail, le comportement en humidité des matériaux hygroscopiques a été démontré à court terme. Il serait intéressant de caractériser ce comportement en humidité dans les mêmes conditions lors d'un essai à plus long terme sur une paroi contenant des matériaux hygroscopiques afin d'identifier le comportement de la paroi en conditions hivernales.

Remerciements. La construction de la double enceinte climatique a été financée par le Fonds Européen de Développement Régional (FEDER) que nous tenons à remercier. Nous adressons également nos remerciements à l'Agence Nationale de la Recherche (ANR) qui a supporté ce travail à travers le projet ANR TransBatiBois (ANR-07-PBAT-008).

\section{Références}

[1] S. Hameury, Moisture buffering capacity of heavy timber structures directly exposed to an indoor climate: a numerical study, Build. Environment 40 (2005) 1400-1412

[2] H.M. Kunzel, A. Holm, K. Sedlbauer, F. Antretter, M. Ellinger, Moisture buffering effect of interior linings made from wood or wood based products, Fraunhofer-Institute for Building Physics, IBP Report HTB-04/2004/e, 2004
[3] O.F. Osanyintola, P. Talukdar, C.J. Simonson, Effect of initial conditions, boundary conditions and thickness on the moisture buffering capacity of spruce plywood, Energy Build. 38 (2006) 1283-1292

[4] O.F. Osanyintola, C.J. Simonson, Moisture buffering capacity of hygroscopic building materials: Experimental facilities and energy impact, Energy Build. 38 (2006) $1270-1282$

[5] C.J. Simonson, M. Salonvaara, T. Ojanen, Moderating indoor conditions with hygroscopic building materials and outdoor ventilation, ASHRAE NA 110 (2004) 804-819

[6] C. James, C.J. Simonson, P. Talukdar, S. Roels, Numerical and experimental data set for benchmarking hygroscopic buffering models, Int. J. Heat Mass Transf. 53 (2010) 3638-3654

[7] D. Medjelekh, S. Abdou, M. El Ganaoui, Impact of the thermal inertia of material on the hygrothermal comfort of building, Int. Rev. Chem. Eng. 2 (2010) 391-397

[8] H. Asan, Effects of wall's insulation thickness and position on time lag and decrement factor, Energy Build. 28 (1998) 299-305

[9] H. Asan, Numerical computation of time lags and decrement factors for different building materials, Build. Environment 41 (2006) 615-620

[10] K. Ghazi Wakili, Ch. Tanner, U-value of a dried wall made of perforated porous clay bricks - Hot box measurement versus numerical analysis, Energy Build. 35 (2003) $675-680$

[11] T. Nussbaumer, K. Ghazi Wakili, Ch. Tanner, Experimental and numerical investigation of the thermal performance of a protected vacuum insulation system applied to a concrete wall, Appl. Energy 83 (2006) 841-855

[12] J. Rose, S. Svendsen, Validating numerical calculations against guarded hot box measurements, Nordic J. Build. Phys. 4 (2004) 9

[13] T. Kalamees, J. Vinha, Hygrothermal calculations and laboratory tests on timber-framed wall structures, Build. Environment 38 (2003) 689-697

[14] Z. Pavlik, R. Cerny, Experimental assessment of hygrothermal performance of an interior thermal insulation system using a laboratory technique simulating on-site conditions, Energy Build. 40 (2008) 673-678

[15] NF EN ISO 8990, Isolation thermique - Détermination des propriétés de transmission thermique en régime stationnaire, Méthodes à la boîte chaude gardée et calibrée, 2006 